Tropical Journal of Pharmaceutical Research December 2020; 19 (12): 2505-2512

ISSN: $1596-5996$ (print); 1596-9827 (electronic)

(C) Pharmacotherapy Group, Faculty of Pharmacy, University of Benin, Benin City, 300001 Nigeria

Available online at http://www.tjpr.org

Original Research Article

http://dx.doi.org/10.4314/tjpr.v19i12.4

\title{
MiR-384-3p aggravates propofol induced apoptosis in developing neurons by targeting Wnt3a
}

\author{
Xiaoling Shi, Wei Jia* \\ Department of Anesthesiology, Hubei Huangshi Central Hospital, Huangshi City, Hubei Province 435000, China
}

*For correspondence: Email: cvm09U@163.com; Tel: +86-7143062022

Sent for review: 8 September 2020

Revised accepted: 24 November 2020

\begin{abstract}
Purpose: To evaluate the cytotoxicity of miR-384-3p toward propofol-treated neonatal rat hippocampal neurons and investigate its related molecular mechanism(s).

Methods: Quantitative real-time polymerase chain reaction ( $q R T-P C R$ ) was employed to determine miR-384-3p expression levels in propofol-treated neonatal rat hippocampal neurons. Cell apoptosis and cell viability were evaluated by flow cytometry analysis and 3-(4,5-dimethylthiazol-2-yl)-2,5diphenyltetrazolium bromide (MTT) assay, respectively. Bioinformatics and dual-luciferase reporter assay were applied together to forecast and verify the interaction between miR-384-3p and Wnt3a. Wnt3a expression in transfected neonatal rat hippocampal neurons was assessed by Western blot assay.

Results: Propofol induced apoptosis in developing neurons by upregulating miR-384-3p expression levels. Knockdown of miR-384-3p reduced propofol-induced apoptosis in developing neurons. Subsequent experiments indicated that miR-384-3p directly regulated Wnt3a expression via coupling with the 3'-untranslated region of Wnt3a. Furthermore, upregulation of Wnt3a expression levels alleviated propofol-induced cytotoxicity promoted by miR-384-3p $(p<0.01)$.

Conclusion: MiR-384-3p aggravates propofol-induced apoptosis in developing neurons by targeting Wnt3a.
\end{abstract}

Keywords: MiR-384-3p, Wnt3a, Propofol, Apoptosis, Developing neurons

\begin{abstract}
This is an Open Access article that uses a funding model which does not charge readers or their institutions for access and distributed under the terms of the Creative Commons Attribution License (http://creativecommons.org/licenses/by/4.0) and the Budapest Open Access Initiative (http://www.budapestopenaccessinitiative.org/read), which permit unrestricted use, distribution, and reproduction in any medium, provided the original work is properly credited.
\end{abstract}

Tropical Journal of Pharmaceutical Research is indexed by Science Citation Index (SciSearch), Scopus, International Pharmaceutical Abstract, Chemical Abstracts, Embase, Index Copernicus, EBSCO, African Index Medicus, JournalSeek, Journal Citation Reports/Science Edition, Directory of Open Access Journals (DOAJ), African Journal Online, Bioline International, Open-J-Gate and Pharmacy Abstracts

\section{INTRODUCTION}

Propofol, an intravenous anesthetic agent, is widely applied in clinical medicine for the induction and maintenance of anesthesia and sedation in children with a rapid onset and short recovery time [1]. However, accumulating evidence has shown that the neurotoxic effects of propofol has raised concerns regarding its safety in pediatric anesthesia [2]. Several biomechanisms of propofol exposure-mediated neurotoxicity have been reported, including neuroinflammation, mitochondrial fission, dysregulation of neurotrophic protein expression, and abnormal regulation of calcium [3]. However, the biomechanism of propofol exposuremediated neurotoxicity toward developing neurons remains unknown. Therefore, we must determine the actual effects of propofol on developing neurons and seek the biomarker to 
avert and minimize any propofol exposuremediated neurotoxicity.

MicroRNAs are implicated in the regulation of diverse cellular functions, including cell proliferation, apoptosis adhesion and invasion by suppressing target mRNA expression at the posttranscriptional level [4]. Studies have shown that miR-384 is an important neuroregulatory factor involved in the survival of neurons. For example, Liu et al. reported that the expression level of miR-384 was upregulated in HT22 cells and hippocampal tissue induced by chronic cerebral ischemia [5]. However, the role of miR-384-3p in neurons remains unclear, and its effect on propofol exposure-induced developmental neurotoxicity needs further study.

Recently, accumulating evidence has reported that the Wnt signaling pathway plays a vital role in neural development, axonal guidance, and neuronal survival [6]. Wingless-type MMTV integration site family, member $3 a$ (Wnt3a), one of the Wnt family members, participates in multiple biological processes, including cell proliferation, self-renewal, motility, and differentiation [7]. Previously, Fragoso et al found that Wnt3a protects retinal ganglion cells from apoptosis [8]. However, whether Wnt3a helps neurons resist propofol exposure-mediated developmental neurotoxicity remains unclear.

In the present study, the expression level of miR384-3p in propofol-treated neonatal rat hippocampal neurons was evaluated. Cell viability and cell apoptosis were then examined with miR-384-3p knockdown. Afterward, whether miR-384-3p regulates propofol exposuremediated developmental neurotoxicity by Wnt3a was investigated. These results indicate an advanced alternative target for the treatment of propofol exposure-mediated developmental neurotoxicity.

\section{EXPERIMENTAL}

\section{Neonatal rat hippocampal neuron isolation and culture}

All animal experiments in this study were conducted in accordance with the Guide for the Care and Use of Laboratory Animals [9] and approved by the Ethics Committee of Hubei Huangshi Central Hospital(Approval no.2018131). Briefly, 2-day-old neonatal SD rats were sacrificed and the whole brains were collected. The hippocampi were separated from the neonatal brains and collected in a tube. The neonatal hippocampal neurons were obtained using collagenase (Sigma-Aldrich, St. Louis, MO,
USA) to digest the hippocampal tissue. The cells were resuspended in culture medium (Neurobasal medium; Gibco, Carlsbad, CA, USA) supplemented with $2 \%$ B27 (Gibco), $1 \%$ GlutaMAX $^{\mathrm{TM}} \quad$ (Gibco) and 1 \% penicillin/streptomycin (Gibco) and cultured in a humidified $5 \% \mathrm{CO}_{2}$ incubator at $37^{\circ} \mathrm{C}$.

\section{Propofol exposure}

Propofol (Sigma-Aldrich) was diluted using dimethyl sulfoxide (DMSO, Sigma-Aldrich) for in vitro assays. The dose of propofol used for humans in clinical settings ranges from approximately 10 to $50 \mu \mathrm{M}$. Specifically, higher doses are applied for anesthesia induction while lower doses are applied for anesthesia maintenance [10]. Thus, neonatal rat hippocampal neurons were exposed to propofol at clinically relevant concentrations $(10,30$, or 50 $\mu \mathrm{M})$ for $6 \mathrm{~h}$. The biomechanism studies were performed in neonatal rat hippocampal neurons following treatment with $30 \mu \mathrm{M}$ propofol for $6 \mathrm{~h}$.

\section{Cell transfection}

Synthetic miR-384-3p mimics, miR-384-3p inhibitor, and miR-control (NC) were acquired from GenePharma (Shanghai, China). After cells were cultured in 6-well plates for $24 \mathrm{~h}$, miR-384$3 p$ mimics, miR-384-3p inhibitor, and miR-control (NC) were transfected into cells using Lipofectamine 2000 (Invitrogen, Carlsbad, CA, USA) according to the manufacturer's instructions.

\section{Quantitative real-time polymerase chain reaction (qRT-PCR)}

Total RNA was extracted from cells using TRIzol reagent (Invitrogen) according to the manufacturer's instructions. The quantity and integrity of the extracted total RNA were measured using a NanoDrop 1000 spectrophotometer (Thermo Fisher Scientific, Inc., Waltham, MA, USA). Total RNA was reverse transcribed into cDNA using the microRNA (miRNA)-specific TaqMan miRNA assay kit (Applied Biosystems, Foster City, CA, USA). QRT-PCR was performed using SYBR Premix EX Taq (Takara, Dalian, China) in the CFX96 Real-Time PCR Detection System (BioRad, Hercules, CA, USA) to examine the expression levels of miR-384-3p. The PCR primers were designed and synthesized by Sangon Biological Engineering Technology (Shanghai, China). U6 small nuclear RNA and $\beta$ actin were used as endogenous reference genes to normalize miRNA and mRNA expression levels, respectively. The relative expression of 
miR-384-3p and Wnt3a in each experimental group was analyzed using the $2^{-\triangle \Delta \mathrm{Ct}}$ method. All the reactions were performed in triplicate. The primer sequences used in this study are shown in Table 1.

\section{3-(4,5-Dimethylthiazol-2-yl)-2,5-diphenyl- tetrazolium bromide (MTT) assay}

The MTT assay was used to examine the extent of cell viability. Briefly, the cells were cultured $(25,000 \mathrm{cell} / \mathrm{mL})$ in 96 -well plates and incubated with $0.5 \mathrm{mg} / \mathrm{mL}$ of MTT solution (Beyotime, Shanghai, China) in culture medium for $4 \mathrm{~h}$ at 37 ${ }^{\circ} \mathrm{C}$. The absorbance value was measured three times using a spectrophotometer (BioTek, Winooski, VT, USA) at a wavelength of $490 \mathrm{~nm}$.

\section{Cell apoptosis}

Cell apoptosis was examined using the Annexin V/FITC and PI Apoptosis Detection kit (Invitrogen) and flow cytometry (BD Accuri ${ }^{\mathrm{MM}} \mathrm{C6}$, BD Biosciences, San Jose, CA, USA). Briefly, the cells were digested, washed, and resuspended in Annexin $\mathrm{V}$ incubation solution. Next, the cells were stained with Annexin Vfluorescein isothiocyanate (FITC) and propidium iodide (PI). The cells were then cultured in the dark for $15 \mathrm{~min}$ at room temperature and quantified using flow cytometric analysis.

\section{Dual-luciferase reporter assay}

The fragments of the 3'-UTRs of Wnt3a mRNA containing the wild-type (WT) and mutant-type (MUT) binding sites of miR-384-3p predicted using starBase were cloned into the pmirGLO luciferase reporter vector (Promega, Madison, WI, USA) to generate the Wnt3a-WT and Wnt3aMUT reporter plasmids, respectively. For the luciferase assay, miR-384-3p mimics or miRcontrol (NC) was co-transfected with the reporter plasmids into cells using Lipofectamine 2000
(Invitrogen). Luciferase activity was measured using the dual-luciferase kit (Promega) after $48 \mathrm{~h}$ transfection according to the manufacturer's protocol. The data were presented as the ratio of experimental (Renilla) luciferase to control (Firefly) luciferase.

\section{Western blot analysis}

Western blot analysis was performed to detect the expression levels of the proteins of interest. Cells were washed three times in cold PBS buffer, and the total protein was extracted using RIPA buffer (Beyotime). The protein concentration was evaluated using the BCA protein assay kit (CoWin Biotechnology, Beijing, China). Equal amounts of total proteins were electrophoresed and then transferred to the polyvinylidene difluoride membranes (PVDF; Millipore, Burlington, MA, USA). Next, the PVDF membranes were blocked with $5 \%$ non-fat milk for $1 \mathrm{~h}$ at room temperature and the proteins were incubated with specific primary antibodies (Rabbit Anti-Wnt3a antibody; ab231178; 1:3000; Abcam, Cambridge, UK) and $\beta$-actin (Rabbit Anti-beta Actin antibody; ab8227; 1:3,000; Abcam) overnight at $4{ }^{\circ} \mathrm{C}$. The membranes were further incubated with the appropriate horseradish peroxidase (HRP)-conjugated goat anti-rabbit immunoglobulin $\mathrm{G}$ secondary antibody (ab205718; 1:3000; Abcam), and the protein bands were visualized by chemiluminescence using the ECL reagent (Beyotime). Anti- $\beta$-actin was used to normalize the amount of the analyzed samples, and the protein bands were quantified by gray value analysis using ImageJ software (National Institutes of Health).

\section{Statistical analysis}

All the data were presented as the means \pm standard error of the mean of three independent experiments.

Table 1: Primers for miR-384-3p, Wnt3a, and reference genes

\begin{tabular}{lll}
\hline Gene & Primer & Sequence $\left(\mathbf{5}^{\prime} \rightarrow \mathbf{3}^{\prime}\right)$ \\
\hline miR-384-3p & Forward & TTTTAGAAGCGTTCGGCCAAA \\
U6 & Reverse & ACCAGAGTTGCCATTGATAGTGA \\
& Forward & TCCTCCACGACAACCAAACC \\
Wnt3a & Reverse & TCTTTCCCAAAATCCAGACTC \\
& Forward & ATCGAGTTTGGTGGGATGGT \\
wt-Wnt3a 3'-UTR & Reverse & CGCTGTCGTACTTGTCCTTG \\
& Forward & TAGGCGATCGCTCGAGGCACCGGCCGCGGCTCCCC \\
mut-Wnt3a 3'-UTR & Reverse & AATTCCCGGGCTCGAGTTTCGTCTAACTCCGTTGGACAGT \\
& Forward & AATGGTCCGCTTCCTGGAGCCAATGGCCCG \\
\multirow{2}{*}{ ß-actin } & Reverse & AGGAAAGCGGACCATTTCCCGCCATGAGGGGCCAGGAAGG \\
& Forward & GTGACGTTGACATCCGTAAAGA \\
& Reverse & GCCGGACTCATCGTACTCC \\
\hline
\end{tabular}


Comparisons between two groups were performed using Student's t-test. GraphPad Prism 5 (GraphPad Software, Inc., San Diego, CA, USA) was used for analysis. $P<0.01$ (twotailed) was considered statistically significant.

\section{RESULTS}

\section{Propofol induces neonatal rat hippocampal neuron toxicity by upregulating miR-384-3p}

Neonatal rat hippocampal neurons were exposed to various concentrations of propofol and, after treatment, cell viability was assessed using the MTT assay. The results showed that propofol treatment reduced cell viability in a dosedependent manner, with significant inhibitory effects at high doses up to $50 \mu \mathrm{M}$ (Figure $1 \mathrm{~A}$ ). In addition, apoptosis was increased by propofol treatment in a dose-dependent manner (Figure 1 B).

Furthermore, the effects of propofol on miR-384$3 p$ expression in neonatal rat hippocampal neurons were examined. qPCR demonstrated that propofol treatment increased the miR-384-3p level in a dose-dependent manner (Figure $1 \mathrm{C}$ ). The result indicated that miR-384-3p might participate in the regulatory effects of propofolinduced neonatal rat hippocampal neuron toxicity. These results indicate that propofol induces neonatal rat hippocampal neuron toxicity and the expression of miR-384-3p in a dosedependent manner.
A
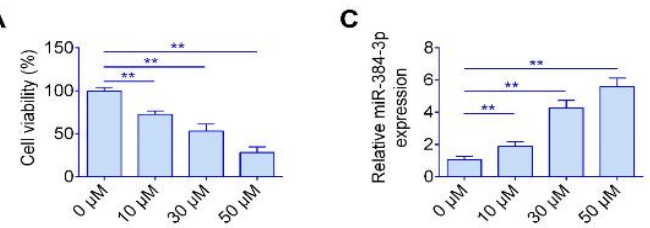

B

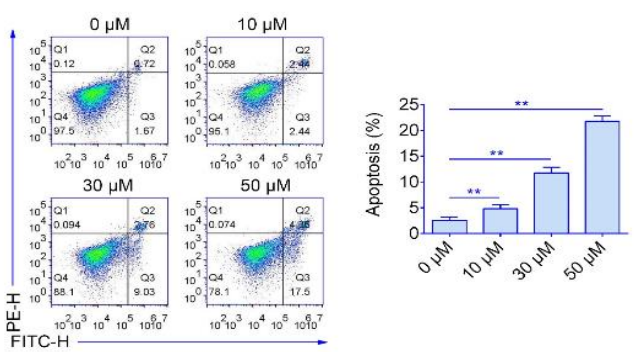

Figure 1: Propofol induces neonatal rat hippocampal neuron toxicity by upregulating miR-384-3p. A) MTT assay was applied to determine the cell viability of each group. B) Flow cytometry analysis was applied to examine apoptosis in each group. C) The miR-384-3p level was measured using qPCR in each group. The data are presented as the means \pm SD of three independent experiments; ${ }^{* *} p<0.01$
Knockdown of miR-384-3p reduces propofolinduced neonatal rat hippocampal neuron toxicity

To evaluate the role of miR-384-3p in propofolmediated neonatal rat hippocampal neuron toxicity, neonatal rat hippocampal neurons were transfected with miR-384-3p inhibitor. After transfection with miR-384-3p inhibitor with or without propofol exposure, the miR-384-3p level in cells transfected with miR-384-3p inhibitor was strikingly lower than that in cells transfected with $\mathrm{NC}$, indicating that cell transfection effectively downregulated miR-384-3p expression (Figure 2 A). The result demonstrated that miR-384-3p inhibitor transfection inhibited propofol-induced miR-384-3p upregulation. Next, cell viability was assessed in each group, and the result demonstrated that the knockdown of miR-384-3p abolished the effect of propofol on cell viability (Figure 2 B). Additionally, cell apoptosis showed that the effects of propofol on neonatal rat hippocampal neurons were remarkably reversed by miR-384-3p inhibition, as evidenced by decreased apoptotic cells with miR-384-3p inhibitor transfection (Figure $2 \mathrm{C}$ ). These results indicate that propofol-induced neonatal rat hippocampal neuron toxicity is reversed by upregulating the level of miR-384-3p.
A

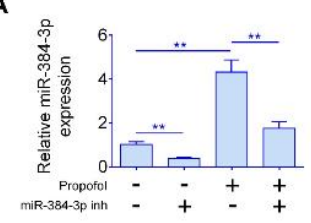

C

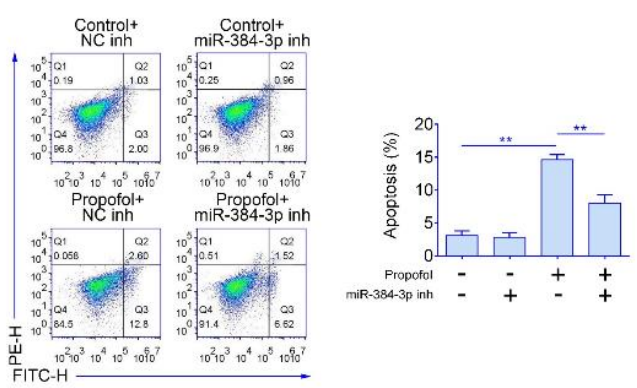

Figure 2: Knockdown of miR-384-3p reduces propofol-induced neonatal rat hippocampal neuron toxicity. A) The miR-384-3p level was measured using real-time PCR. Cells were divided into four groups, NC, miR-384-3p inhibitor, propofol + NC, and propofol + miR-384-3p inhibitor. The dose and duration of treatments were $6 \mu \mathrm{M}$ and $48 \mathrm{~h}$, respectively. B) The MTT assay was applied to determine the cell viability of each group. C) Flow cytometry analysis was applied to detect apoptosis of each group. The data are presented as the means $\pm S D$ of three independent experiments; ${ }^{* *} p<0.01$ 


\section{MiR-384-3p directly regulates Wnt3a expression by binding to the 3'-UTR of Wnt3a}

To further evaluate the biomechanism of miR384-3p in neonatal rat hippocampal neurons, the mRNA binding sites were predicted in starBase (http://starbase.sysu.edu.cn/). The results indicated that Wnt3a mRNA is a binding target of miR-384-3p. The predicted 3'-UTRs of Wnt3a mRNA binding to miR-384-3p are presented in Figure $3 \mathrm{~A}$. To investigate whether Wnt3a was a potential target of miR-384-3p, Wnt3a WT and MUT fragments were cloned downstream of the firefly luciferase coding region. The overexpression of miR-384-3p strikingly reduced the luciferase activity of the Wnt3a-WT reporter gene but did not affect the Wnt3a-MUT control (Figure $3 \mathrm{~B}$ ). To further verify whether miR-384$3 p$ regulated Wnt3a expression, miR-384-3p inhibitor or control was transfected into neonatal rat hippocampal neurons to examine the expression levels of Wnt3a proteins following propofol exposure. Western blotting showed that high expression of miR-384-3p induced by propofol exposure significantly suppressed Wnt3a protein expression. However, miR-384-3p inhibitor significantly promoted Wnt3a mRNA expression compared with that in the inh-NC group following propofol exposure (Figure $3 \mathrm{C}$ ). The data show that Wnt3a is a target of miR-384$3 p$ and miR-384-3p represses the Wnt3a level.

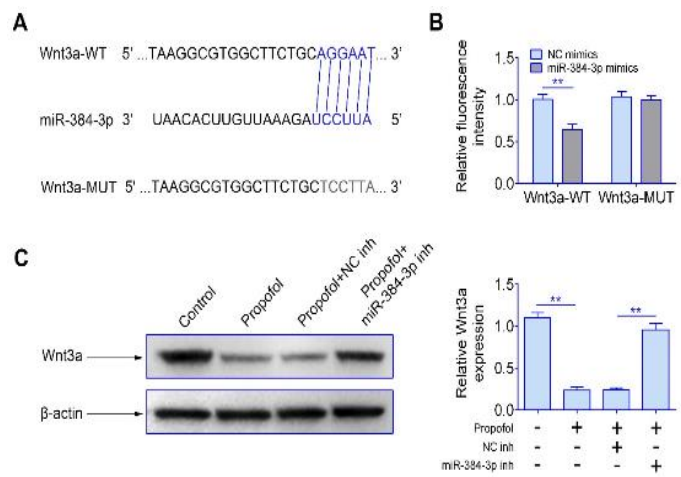

Figure 3: MiR-384-3p directly regulates Wnt3a expression by binding to the $3^{\prime}-$ UTR of Wnt3a. A) Predicted miR-384-3p binding sites on the Wnt3a target gene by starBase. B) Dual-luciferase assays were performed after cells were co-transfected Wnt3aWT or Wnt3a-MUT with miR-384-3p mimics or miRcontrol for $48 \mathrm{~h}$. C) The expression level of Wnt3a protein in cells transfected with miR-384-3p inhibitor or control following propofol exposure was determined by western blotting. The Wnt3a protein expression level in cells without propofol exposure served as a negative control. $\beta$-Actin was applied as the endogenous reference gene. The data are presented as the means $\pm S D$ of three independent experiments; ${ }^{* *} p<0.01$ Upregulation of Wnt3a alleviates propofol-
induced cytotoxicity

To determine whether propofol influence neonatal rat hippocampal neuron toxicity by regulating Wnt3a expression, a rescue experiment was performed. The neonatal rat hippocampal neurons were transfected with either vector control or Wnt3a overexpressing vector with either miR-384-3p mimics or miRcontrol. Western blot analysis confirmed that miR-384-3p mimics obviously suppressed the expression of Wnt3a in neonatal rat hippocampal neurons while Wnt3a overexpression vector restored the expression levels of Wnt3a proteins by significantly promoting the expression of Wnt3a in neonatal rat hippocampal neurons (Figure $4 \mathrm{~A}$ ).

To further verify whether Wnt3a alleviates propofol-induced cytotoxicity promoted by miR$384-3 p$, cell viability and apoptosis were assessed in each group. Cell viability analysis indicated that propofol-mediated, miR-384-3prepressed cell viability was abrogated by Wnt3a overexpression (Figure 4 B). Similarly, overexpression of Wnt3a also abolished the effect of propofol-induced, miR-384-3p-promoted apoptosis (Figure $4 \mathrm{C}$ ). Taken together, these results suggest that the effect of propofol on neonatal rat hippocampal neuron toxicity is mediated by the miR-384-3p/Wnt3a axis.

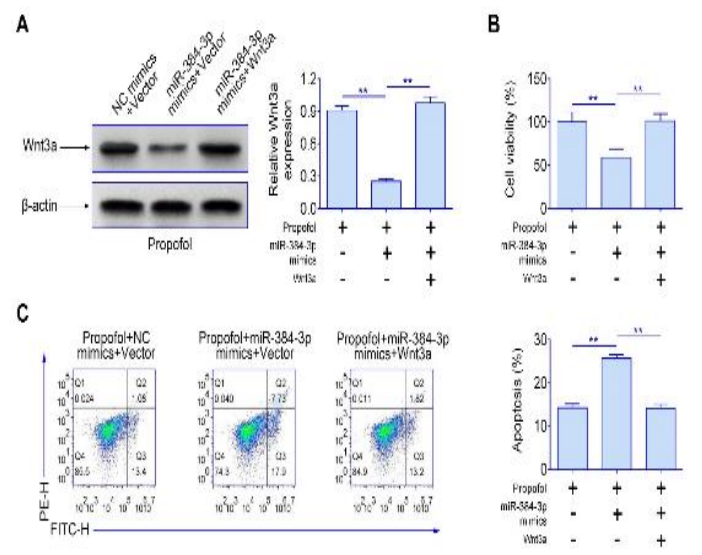

Figure 4: Upregulation of Wnt3a alleviates propofolinduced cytotoxicity promoted by miR-384-3p. (A) The levels of Wnt3a protein in cells transfected with either vector control or Wnt3a-overexpressing vector with either miR-384-3p mimics or miR-control with propofol exposure as determined by western blotting. $\beta$-Actin was applied as the endogenous reference genes. (B) The MTT assay was performed to determine the cell viability of each group. (C) Flow cytometry analysis was applied to detect apoptosis in each group. Data were presented as the mean $\pm S D$ with three independent experiments; ${ }^{* *} p<0.01$ 


\section{DISCUSSION}

Accumulating evidence indicates that exposure of immature animals to anesthetic agents induces the degeneration and apoptosis of neurons [11]. Moreover, studies have shown that anesthetic drugs lead to learning disabilities later in life in animals exposed to anesthetic drugs early in life [12]. Specifically, studies performed in primate models have shown that propofol induced neuron toxicity with exposure for 5 to $6 \mathrm{~h}$ [13]. For example, $5 \mathrm{~h}$ of exposure to propofol was sufficient to maintain the level of surgical anesthesia and induce significant neuronal apoptosis in neonatal rhesus macaques [14]. Additionally, cultured neonatal rat hippocampal neurons died when exposed to 5 and $50 \mu \mathrm{M}$ propofol for $5 \mathrm{~h}$ [15].

However, the mechanism by which propofol neurotoxicity occurs in developing neurons remains unexplained. In this study, propofol treatment reduced neonatal rat hippocampal neuron viability in a dose-dependent manner, with significant inhibitory effects at high doses. In addition, apoptosis was increased by propofol treatment in a dose-dependent manner.

MiRNAs have been shown to play vital roles in regulating disease processes and homeostasis [16]. Many miRNAs are highly expressed in the nervous system, contributing to the maintenance of normal neuronal functions and homeostasis, and affecting neurogenesis [17]. Moreover, many miRNAs are implicated in neurological diseases and disorders of miRNAs play a vital role in neurotoxicity [18]. Specifically, studies have also found that propofol significantly changes the expression level of miRNAs in primary embryonic neural stem cells or astrocytes of immature rats, and -miRNAs regulated by propofol are specifically associated with cell apoptosis [19].

Recently, miR-34a was significantly increased in SH-SY5Y cells treated with propofol [20]. Moreover, miR-665 was overexpressed after propofol treatment, and miR-665 knockdown inhibited propofol exposure-mediated apoptosis [21]. However, the mechanism of propofolregulated miRNAs requires further investigation. This study revealed that miR-384-3p was upregulated in propofol-treated neonatal rat hippocampal neurons in a dose-dependent manner, and propofol-induced neonatal rat hippocampal neuron toxicity was reversed by upregulating the level of miR-384-3p. These results hint that miR-384-3p may act as a promoting factor in propofol-mediated apoptosis in neonatal rat hippocampal neurons.
An increasing number of studies have confirmed that miRNAs perform their functions by regulating the expression of target mRNAs [22]. miR-384$5 p$ participated in neuronal apoptosis induced by rotenone via targeting glucose-regulated protein 78 (GRP78) [23]. Moreover, mechanically injured PC12 cells were involved in activating the miR384-5p-upregulated promoter of the autophagy (Beclin-1) signaling pathway [24]. In addition, anesthesia-induced damage of abalone basal ganglia neurons was mediated by the miR-15a5p/ACVR1 pathway[25]. Another novel discovery of this study is that Wnt3a is a target of miR-384$3 p$. Wnt3a belongs to a gene family encoding secreted signaling proteins involved in several developmental processes.

This study demonstrated that miR-384-3p targeted Wnt3a by predicting the mRNA binding sites in starBase and performing dual-luciferase assays. Moreover, high expression of miR-384$3 p$ induced by propofol exposure significantly suppressed Wnt3a protein expression. Furthermore, propofol-mediated miR-384-3p repressed cell viability, an effect that was abrogated by Wnt3a overexpression.

Similarly, overexpression of Wnt3a also abolished the effect of propofol-induced, miR384-3p-promoted apoptosis. The data indicated that Wnt3a alleviated propofol-induced cytotoxicity promoted by miR-384-3p.

\section{CONCLUSION}

Upregulation of miR-384-3p and downregulation of Wnt3a are mediated by propofol exposure. Moreover, knockdown of miR-384-3p decreases propofol-mediated apoptosis and cell viability in neonatal rat hippocampal neurons by targeting Wnt3a. Moreover, overexpression of Wnt3a reverses the effect induced by propofol exposure. This study revealed the role of miR384-3p/Wnt3a in propofol-mediated neurotoxicity, paving the path for advanced therapeutic targets in developing neurons.

\section{DECLARATIONS}

\section{Conflict of interest}

No conflict of interest is associated with this work.

\section{Contribution of authors}

We declare that this work was done by the authors named in this article and all liabilities pertaining to claims relating to the content of this 
article will be borne by the authors. XS and WJ designed the study, supervised the data collection, analyzed the data, interpreted the data and prepared the manuscript for publication, supervised the data collection, analyzed the data and reviewed the draft of the manuscript. All authors have read and approved the manuscript.

\section{Availability of data and materials}

All data generated or analyzed during this study are included in this published article.

\section{Open Access}

This is an Open Access article that uses a funding model which does not charge readers or their institutions for access and distributed under the terms of the Creative Commons Attribution License (http://creativecommons.org/licenses/by/ 4.0) and the Budapest Open Access Initiative (http://www.budapestopenaccessinitiative.org/rea d), which permit unrestricted use, distribution, and reproduction in any medium, provided the original work is properly credited.

\section{REFERENCES}

1. Chidambaran V, Costandi A, D'Mello A. Propofol: a review of its role in pediatric anesthesia and sedation. CNS Drugs 2015; 29(7): 543-563.

2. Li J-K, Liu X-D, Shen L, Zeng W-M, Qiu G-Z. Natural plant polyphenols for alleviating oxidative damage in man: Current status and future perspectives. Trop $J$ Pharm Res 2016; 15(5): 1089-1098.

3. Wei $\mathrm{H}$. The role of calcium dysregulation in anestheticmediated neurotoxicity. Anesth Analg 2011; 113(5): 972974.

4. Bartel DP. MicroRNAs: genomics, biogenesis, mechanism, and function. Cell 2004; 116(2): 281-297.

5. Liu J, An P, Xue Y, Che D, Liu X, Zheng J, Liu Y, Yang C, Li Z, Yu B. Mechanism of Snhg8/miR384/Hoxa13/FAM3A axis regulating neuronal apoptosis in ischemic mice model. Cell Death Dis 2019; 10(6): 441.

6. González-Fernández C, Fernández-Martos CM, Shields $S D$, Arenas E, Javier Rodríguez F. Whts are expressed in the spinal cord of adult mice and are differentially induced after injury. J Neurotrauma 2014; 31(6): 565581.

7. He S, Lu Y, Liu X, Huang X, Keller ET, Qian CN, Zhang $J$ J. Wnt3a: functions and implications in cancer. Chin J Cancer 2015; 34(12): 554-562.

8. Fragoso MA, Yi H, Nakamura RE, Hackam AS. The Wnt signaling pathway protects retinal ganglion cell 5 (RGC5) cells from elevated pressure. Cell Mol Neurobiol 2011; 31(1): 163-173.
9. National Research Council Committee for the Update of the Guide for the C, Use of Laboratory A: The National Academies Collection: Reports funded by National Institutes of Health. In: Guide for the Care and Use of Laboratory Animals. Washington (DC): National Academies Press (US) Copyright (c) 2011, National Academy of Sciences; 2011.

10. Twaroski DM, Yan Y, Olson JM, Bosnjak ZJ, Bai X. Down-regulation of microRNA-21 is involved in the propofol-induced neurotoxicity observed in human stem cell-derived neurons. Anesthesiology 2014; 121(4): 786800.

11. Olney JW, Wozniak DF, Jevtovic-Todorovic V, Farber NB, Bittigau P, Ikonomidou C. Drug-induced apoptotic neurodegeneration in the developing brain. Brain Pathol 2002; 12(4): 488-498.

12. Jevtovic-Todorovic V, Hartman RE, Izumi $Y$, Benshoff ND, Dikranian K, Zorumski CF, Olney JW, Wozniak DF. Early exposure to common anesthetic agents causes widespread neurodegeneration in the developing rat brain and persistent learning deficits. J Neurosci 2003; 23(3): 876-882.

13. McCann ME, Soriano SG. Does general anesthesia affect neurodevelopment in infants and children? Bmj 2019; 367: 16459.

14. Creeley C, Dikranian K, Dissen G, Martin L, Olney J, Brambrink A. Propofol-induced apoptosis of neurones and oligodendrocytes in fetal and neonatal rhesus macaque brain. Br J Anaesth 2013; 110: 29-38.

15. Kahraman S, Zup SL, McCarthy MM, Fiskum G. GABAergic mechanism of propofol toxicity in immature neurons. J Neurosurg Anesthesiol 2008; 20(4): 233-240.

16. Hu Y, Liu Q, Zhang M, Yan Y, Yu H, Ge L. MicroRNA362-3p attenuates motor deficit following spinal cord injury via targeting paired box gene 2. J Integr Neurosci 2019; 18(1): 57-64.

17. Krichevsky AM, King KS, Donahue CP, Khrapko K, Kosik KS. A microRNA array reveals extensive regulation of microRNAs during brain development. Rna 2003; 9(10): 1274-1281.

18. Johnson R, Zuccato C, Belyaev ND, Guest DJ, Cattaneo E, Buckley NJ. A microRNA-based gene dysregulation pathway in Huntington's disease. Neurobiol Dis 2008; 29(3): 438-445.

19. Sun W, Pei L. microRNA Expression Profiling of PropofolTreated Developing Rat Hippocampal Astrocytes. DNA Cell Biol 2015; 34(8): 511-523.

20. Li GF, Li ZB, Zhuang SJ, Li GC. Inhibition of microRNA$34 a$ protects against propofol anesthesia-induced neurotoxicity and cognitive dysfunction via the MAPKJERK signaling pathway. Neurosci Lett 2018; 675: 152-159.

21. Sun WC, Pei L. rno-miR-665 targets BCL2L1 (BCl-xl) and increases vulnerability to propofol in developing astrocytes. J Neurochem 2016; 138(2): 233-242.

22. Bartel DP. MicroRNAs: target recognition and regulatory functions. Cell 2009; 136(2): 215-233. 
23. Jiang $M$, Yun $Q$, Shi F, Niu G, Gao Y, Xie S, Yu S. Downregulation of miR-384-5p attenuates rotenoneinduced neurotoxicity in dopaminergic SH-SY5Y cells through inhibiting endoplasmic reticulum stress. Am J Physiol Cell Physiol 2016; 310(9): C755-763.

24. Zhou Z, Hu B, Lyu Q, Xie T, Wang J, Cai Q. miR-384-5p promotes spinal cord injury recovery in rats through suppressing of autophagy and endoplasmic reticulum stress. Neurosci Lett 2020; 727: 134937.

25. Wang Z, Du X, Yang Y, Zhang G. Study on miR-384-5p activates TGF- $\beta$ signaling pathway to promote neuronal damage in abutment nucleus of rats based on deep learning. Artif Intell Med 2019; 101: 101740. 